\title{
Hardy Inequalities \\ for Overdetermined Classes of Functions
}

\author{
A. Kufner and C. G. Simader
}

Abstract. Conditions on weights $w_{0}$ and $w_{k}$ are given for the $k$-th order Hardy inequality $\left(\int_{0}^{1}|u(t)|^{q} w_{0}(t) d t\right)^{1 / q} \leq c\left(\int_{0}^{1}\left|u^{(k)}(t)\right|^{p} w_{k}(t) d t\right)^{1 / p}$ to hold for two special classes of functions $u$ satisfying $2 k$ and $k+1$ boundary conditions, respectively. The conditions are sufficient and partially also necessary. For one class, a hypothesis is formulated describing necessary and sufficient conditions on $w_{0}$ and $w_{k}$.

Keywords: Hardy's inequality, weighted norm inequalities

AMS subject classification: $26 \mathrm{D} 10$

\section{Introduction}

The $k$-th order Hardy inequality

$$
\left(\int_{0}^{1}|u(t)|^{q} w_{0}(t) d t\right)^{\frac{1}{q}} \leq c\left(\int_{0}^{1}\left|u^{(k)}(t)\right|^{p} w_{k}(t) d t\right)^{\frac{1}{p}}
$$

with parameters $p, q(0<q \leq \infty, 1 \leq p \leq \infty)$ and weight functions $w_{0}, w_{k}$ (i.e., functions measurable and positive a.e. in $(0,1))$ is meaningful if we consider classes of functions $u$ satisfying certain boundary conditions

$$
\left.\begin{array}{ll}
u^{(i)}(0)=0 & \text { for } i \in M_{0} \\
u^{(j)}(1)=0 & \text { for } j \in M_{1}
\end{array}\right\}
$$

with $M_{0}, M_{1} \subset\{0,1, \ldots, k-1\}$. It is reasonable to consider sets $M_{0}$ and $M_{1}$ such that

$$
\# M_{0}+\# M_{1}=k \text {; }
$$

the admissible sets $M_{0}, M_{1}$ are described in $[1,2,4]$.

The paper deals with the problem of finding conditions (sufficient, or necessary and sufficient) if the number of boundary conditions exceeds $k$. Such classes of functions will be called overdetermined.

A. Kufner: Czech Academy of Sciences, Mathematical Institute, Žitná 25, 11567 Praha 1, Czech Republic

C. G. Simader: Universität Bayreuth, Fakultät für Mathematik, D - 95440 Bayreuth, Germany 
For the case $k=1$, the only overdetermined class is that of functions satisfying

$$
u(0)=u(1)=0
$$

and the necessary and sufficient condition for (1) to hold for such functions has for the case

$$
1<p \leq q<\infty
$$

the following form:

$$
\sup _{0<a<b<1}\left(\int_{a}^{b} w_{0}(t) d t\right)^{\frac{1}{q}} \min \left\{\left(\int_{0}^{a} w_{1}^{1-p^{\prime}}(t) d t\right)^{\frac{1}{p^{\prime}}},\left(\int_{b}^{1} w_{1}^{1-p^{\prime}}(t) d t\right)^{\frac{1}{p^{\prime}}}\right\}<\infty
$$

(see [3: Section 8]). For $k \in \mathbb{N}, k>1$, we have more possibilities concerning the choice of overdetermined classes. Here, we will deal with two special cases:

Case I. We will consider the maximal class of overdetermined functions, i.e., functions, satisfying the maximal number of conditions:

$$
\left.\begin{array}{l}
u^{(i)}(0)=0 \\
u^{(i)}(1)=0
\end{array}\right\} \quad(i=0,1, \ldots, k-1) .
$$

Here $M_{0}=M_{1}=\{0,1, \ldots, k-1\}$ and $\# M_{0}+\# M_{1}=2 k$.

Case II. We will consider the class of functions satisfying

$$
\left.\begin{array}{rl}
u^{(i)}(0) & =0 \quad(i=0,1, \ldots, k-1) \\
u^{(k-1)}(1) & =0
\end{array}\right\}
$$

i.e., $M_{0}=\{0,1, \cdots, k-1\}, M_{1}=\{k-1\}$ and $\# M_{0}+\# M_{1}=k+1$.

\section{The Case I}

The inequality (1) holds for all functions $u$ satisfying the conditions

$$
u(0)=u^{\prime}(0)=\ldots=u^{(k-1)}(0)=0
$$

or

$$
u(1)=u^{\prime}(1)=\ldots=u^{(k-1)}(1)=0
$$

if and only if the functions

$$
\begin{aligned}
& B_{1}(x)=\left(\int_{x}^{1}(t-x)^{(k-1) q} w_{0}(t) d t\right)^{\frac{1}{q}}\left(\int_{0}^{x} w_{k}^{1-p^{\prime}}(t) d t\right)^{\frac{1}{p^{\prime}}} \\
& B_{2}(x)=\left(\int_{x}^{1} w_{0}(t) d t\right)^{\frac{1}{q}}\left(\int_{0}^{x}(x-t)^{(k-1) p^{\prime}} w_{k}^{1-p^{\prime}}(t) d t\right)^{\frac{1}{p^{r}}}
\end{aligned}
$$


are bounded on $(0,1)$ (in the case (9)) or if and only if the functions

$$
\begin{aligned}
& B_{3}(x)=\left(\int_{0}^{x}(x-t)^{(k-1) q} w_{0}(t) d t\right)^{\frac{1}{q}}\left(\int_{x}^{1} w_{k}^{1-p^{\prime}}(t) d t\right)^{\frac{1}{p^{\prime}}} \\
& B_{4}(x)=\left(\int_{0}^{x} w_{0}(t) d t\right)^{\frac{1}{q}}\left(\int_{x}^{1}(t-x)^{(k-1) p^{\prime}} w_{k}^{q-p^{\prime}}(t) d t\right)^{\frac{1}{p^{\prime}}}
\end{aligned}
$$

are bounded on $(0,1)$ (in the case (10)). [Let us recall that throughout the paper, we assume (5) - i.e. $1<p \leq q<\infty-$ and that $p^{\prime}=\frac{p}{p-1}$ is the number adjoint to p.]

Consequently, we can conclude that for functions satisfying (7) - i.e., (9) and (10) simultaneously - the boundedness of the pair of functions $B_{1}, B_{2}$ or the boundedness of the pair of functions $B_{3}, B_{4}$ is a sufficient condition for the validity of inequality (1).

But we can proceed in another way: The inequality (1) holds for all functions satisfying the conditions

$$
\left.\begin{array}{rl}
u(0)=u^{\prime}(0)=\cdots=u^{(k-2)}(0) & =0 \\
u^{(k-1)}(1) & =0
\end{array}\right\}
$$

or the conditions

$$
\left.\begin{array}{rl}
u^{(k-1)}(0) & =0 \\
u(1)=u^{\prime}(1)=\cdots=u^{(k-2)}(1) & =0
\end{array}\right\}
$$

if and only if the functions

$$
\begin{aligned}
& \widetilde{B}_{1}(x)=\left(\int_{x}^{1} t^{(k-2) q} w_{0}(t) d t\right)^{\frac{1}{q}}\left(\int_{0}^{x} t^{p^{\prime}} w_{k}^{1-p^{\prime}}(t) d t\right)^{\frac{1}{p^{\prime}}} . \\
& \widetilde{B}_{2}(x)=\left(\int_{0}^{x} t^{(k-1) q} w_{0}(t) d t\right)^{\frac{1}{q}}\left(\int_{x}^{1} w_{k}^{1-p^{\prime}}(t) d t\right)^{\frac{1}{p^{\prime}}}
\end{aligned}
$$

are bounded on $(0,1)$ (in the case (13)) or if and only if the functions

$$
\begin{aligned}
& \widetilde{B}_{3}(x)=\left(\int_{0}^{x}(1-t)^{(k-2) q} w_{0}(t) d t\right)^{\frac{1}{4}}\left(\int_{x}^{1}(1-t)^{p^{\prime}} w_{k}^{1-p^{\prime}}(t) d t\right)^{\frac{1}{p^{\prime}}} \\
& \widetilde{B}_{4}(x)=\left(\int_{x}^{1}(1-t)^{k-1) q} w_{0}(t) d t\right)^{\frac{1}{q}}\left(\int_{0}^{x} w_{k}^{1-p^{\prime}}(t) d t\right)^{\frac{1}{p^{\prime}}}
\end{aligned}
$$

are bounded on $(0,1)$ (in the case (14)). Since the conditions (13) and (14) together indicate that $u$ satisfies conditions $(7)$, the boundedness of the pair of functions $\widetilde{B}_{1}, \widetilde{B}_{2}$ or 
of the pair of functions $\widetilde{B}_{3}, \widetilde{B}_{4}$ is another sufficient condition for the validity of inequality (1) in the Case I.

Using some other combinations of boundary conditions which together guarantee the validity of (7), we can derive also other types of sufficient conditions for (1) to hold. But first, let us formulate a conjecture motivated by the condition (6), which is necessary and sufficient for (1) to hold in the Case I if $k=1$.

Hypothesis. Inequality (1) holds for all functions $u$ satisfying (7) if and only if the conditions

$$
\begin{aligned}
\sup _{0<a<b<1}\left(\int_{a}^{b} w_{0}(t) d t\right)^{\frac{1}{a}} \min & \left\{\left(\int_{0}^{a}(a-t)^{(k-1) p^{\prime}} w_{k}^{1-p^{\prime}}(t) d t\right)^{\frac{1}{p^{\prime}}},\right. \\
& \left.\left(\int_{b}^{1}(t-b)^{(k-1) p^{\prime}} w_{k}^{1-p^{\prime}}(t) d t\right)^{\frac{1}{p^{\prime}}}\right\}<\infty
\end{aligned}
$$

and

$$
\begin{aligned}
\sup _{0<a<b<1} \min & \left\{\left(\int_{0}^{a}(a-t)^{(k-1) q} w_{0}(t) d t\right)^{\frac{1}{a}},\right. \\
& \left.\left(\int_{b}^{1}(t-b)^{(k-1) q} w_{0}(t) d t\right)^{\frac{1}{a}}\right\}\left(\int_{a}^{b} w_{k}^{1-p^{\prime}}(t) d t\right)^{\frac{1}{p^{t}}}<\infty
\end{aligned}
$$

are satisfied.

We will not prove this hypothesis and, instead, some other sufficient conditions will be given. Let us also remark that the conditions connected with the functions $B_{i}$ and concerning functions $u$ satisfying (9) or (10) are due to V. Stepanov while the conditions expressed by the functions $\widetilde{B}_{i}$ and concerning functions $u$ satisfying (13) or (14) are due to H. P. Heinig and A. Kufner. The results are collected, e.g., in [2] or [3].

Theorem 1. Let $1<p \leq q<\infty$. Let $w_{0}$ and $w_{k}$ be weight functions on $(0,1)$ and assume that there exists a constant $C>0$ such that the estimates

$$
\begin{aligned}
& \left(\int_{0}^{a} w_{0}(t) d t\right)^{\frac{1}{q}}\left(\int_{a}^{1} w_{k}^{1-p^{\prime}}(t) d t\right)^{\frac{1}{p^{t}}} \leq C \\
& \left(\int_{b}^{1} w_{0}(t) d t\right)^{\frac{1}{q}}\left(\int_{0}^{b} w_{k}^{1-p^{\prime}}(t) d t\right)^{\frac{1}{p^{\prime}}} \leq C
\end{aligned}
$$




$$
\begin{aligned}
\left(\int_{a}^{b} w_{0}(t) d t\right)^{\frac{1}{q}} \min \{ & \left(\int_{0}^{a}(a-t)^{(k-1) p^{\prime}} w_{k}^{1-p^{\prime}}(t) d t\right)^{\frac{1}{p^{\prime}}}, \\
& \left.\left(\int_{b}^{1}(t-b)^{(k-1) p^{\prime}} w_{k}^{1-p^{\prime}}(t) d t\right)^{\frac{1}{p^{\prime}}}\right\} \leq C
\end{aligned}
$$

hold for every $a, b \in(0,1) \quad(a<b$ in (21)). Then the Hardy inequality (1) holds for every function $u \in A C^{(k-1)}(0,1)$ satisfying the conditions

$$
u^{(i)}(0)=u^{(i)}(1)=0 \quad(i=0,1, \ldots, k-1) .
$$

Remark. Notice that condition (21) is in fact condition (17) in the Hypothesis and coincides with (6) for $k=1$. In fact, we follow in the proof the idea of P. Gurka used for $k=1$ (see, e.g., [3: Section 8]).

Proof of Theorem 1. There is an integer $m \in \mathbb{Z}$ such that

$$
2^{m} \leq \sup _{0<x<1}|u(x)|<2^{m+1}
$$

For $j \in \mathbb{Z}, j \leq m$, let $x_{j}$ and $y_{j}$ be the smallest and greatest numbers from $(0,1)$ such that $\left|u\left(x_{j}\right)\right|=2^{j}$ and $\left|u\left(y_{j}\right)\right|=2^{j}$, respectively. Obviously, $x_{j-1}<x_{j} \leq y_{j}<y_{j-1}$, $(0,1)=\bigcup_{j<m}\left[x_{j-1}, x_{j}\right] \cup\left[x_{m}, y_{m}\right] \bigcup_{j<m}\left[y_{j}, y_{j-1}\right]$ and

$$
\int_{0}^{1}|u(x)|^{q} w_{0}(x) d x \leq \sum_{j \leq m} 2^{(j+1) q} \int_{x_{j}}^{y_{j}} w_{0}(x) d x .
$$

Since $u$ satisfies (22), we have

$$
\begin{aligned}
(k-1) ! u\left(x_{j}\right) & =\int_{0}^{x_{j}}\left(x_{j}-t\right)^{k-1} u^{(k)}(t) d t \\
(k-1) ! u\left(x_{j-1}\right) & =\int_{0}^{x_{j-1}}\left(x_{j-1}-t\right)^{k-1} u^{(k)}(t) d t
\end{aligned}
$$

and

$$
\begin{aligned}
(k-1) ! & \left|u\left(x_{j}\right)-u\left(x_{j-1}\right)\right| \\
& =\left|\int_{0}^{x_{j-1}}\left[\left(x_{j}-t\right)^{k-1}-\left(x_{j-1}-t\right)^{k-1}\right] u^{(k)}(t) d t+\int_{x_{j-1}}^{x_{j}}\left(x_{j}-t\right)^{k-1} u^{(k)}(t) d t\right| \\
& \leq(k-1)\left(x_{j}-x_{j-1}\right) \int_{0}^{x_{j-1}}\left|u^{(k)}(t)\right| d t+\int_{x_{j-1}}^{x_{j}}\left(x_{j}-t\right)^{k-1}\left|u^{(k)}(t)\right| d t .
\end{aligned}
$$


Using the Hölder inequality and the estimate

$$
\left|u\left(x_{j}\right)-u\left(x_{j-1}\right)\right| \geq\left|u\left(x_{j}\right)\right|-\left|u\left(x_{j-1}\right)\right|=2^{j}-2^{j-1}=2^{j-1},
$$

we obtain

$$
\begin{aligned}
2^{j-1} \leq & c\left(x_{j}-x_{j-1}\right)\left(\int_{0}^{x_{j}-1}\left|u^{(k)}(t)\right|^{p} w_{k}(t) d t\right)^{\frac{1}{p}}\left(\int_{0}^{x_{j-1}} w_{k}^{1-p^{\prime}}(t) d t\right)^{\frac{1}{p^{\prime}}} \\
& +c\left(\int_{x_{j-1}}^{x_{j}}\left|u^{(k)}(t)\right|^{p} w_{k}(t) d t\right)^{\frac{1}{p}}\left(\int_{x_{j-1}}^{x_{j}}\left(x_{j}-t\right)^{(k-1) p^{\prime}} w_{k}^{1-p^{\prime}}(t) d t\right)^{\frac{1}{p^{\prime}}}
\end{aligned}
$$

and consequently,

$$
\begin{aligned}
2^{(j-1) q} \int_{x_{j}}^{y_{j}} w_{0}(t) d t \leq & 2^{q-1} c^{q}\left(x_{j}-x_{j-1}\right)^{q}\left(\int_{0}^{x_{j-1}}\left|u^{(k)}(t)\right|^{p} w_{k}(t) d t\right)^{\frac{q}{p}} \\
& \times\left(\int_{x_{j}}^{y_{j}} w_{0}(t) d t\right)\left(\int_{0}^{x_{j-1}} w_{k}^{1-p^{\prime}}(t) d t\right)^{\frac{q}{p^{\prime}}} \\
& +2^{q-1} c^{q}\left(\int_{x_{j-1}}^{x_{j}}\left|u^{(k)}(t)\right|^{p} w_{k}(t) d t\right)^{\frac{q}{p}} \\
& \times\left(\int_{x_{j}}^{y_{j}} w_{0}(t) d t\right)\left(\int_{x_{j-1}}^{x_{j}}\left(x_{j}-t\right)^{(k-1) p^{\prime}} w_{k}^{1-p^{\prime}}(t) d t\right)^{\frac{q}{p^{\prime}}}
\end{aligned}
$$

Moreover, using condition (20) (with $b=x_{j}$ ), the first term on the right-hand side can be estimated by

$$
\begin{gathered}
\left(x_{j}-x_{j-1}\right)^{q}\left(\int_{0}^{1}\left|u^{(k)}(t)\right|^{p} w_{k}(t) d t\right)^{\frac{q}{p}}\left(\int_{x_{j}}^{1} w_{0}(t) d t\right)\left(\int_{0}^{x_{j}} w_{k}^{1-p^{\prime}}(t) d t\right)^{\frac{q}{p^{\prime}}} \\
\leq\left(x_{j}-x_{j-1}\right)^{q}\left(\int_{0}^{1}\left|u^{(k)}(t)\right|^{p} w_{k}(t) d t\right)^{\frac{q}{p}} C^{q}
\end{gathered}
$$

so that we have finally 


$$
\begin{aligned}
2^{(j-1) q} \int_{x_{j}}^{y_{j}} w_{0}(t) d t \leq & 2^{q-1} c^{q} C^{q}\left(x_{j}-x_{j-1}\right)^{q}\left(\int_{0}^{1}\left|u^{(k)}(t)\right|^{p} w_{k}(t) d t\right)^{\frac{q}{p}} \\
& +2^{q-1} c^{q}\left(\int_{x_{j-1}}^{x_{j}}\left|u^{(k)}(t)\right|^{p} w_{k}(t) d t\right)^{\frac{q}{p}} \\
& \times\left(\int_{x_{j}}^{y_{j}} w_{0}(t) d t\right)\left(\int_{x_{j-1}}^{x_{j}}\left(x_{j}-t\right)^{(k-1) p^{\prime}} w_{k}(t) d t\right)^{\frac{q}{p^{\prime}}} .
\end{aligned}
$$

Since $u$ satisfies (22), we have also $(-1)^{k}(k-1) ! u(y)=\int_{y}^{1}(t-y)^{k-1} u^{(k)}(t) d t$. Putting here $y=y_{j}$ and $y=y_{j-1}$, we obtain immediately the following analogy of (24):

$$
\begin{aligned}
2^{(j-1) q} \int_{x_{j}}^{y_{j}} w_{0}(t) d t \leq & 2^{q-1} c^{q}\left[\left(y_{j-1}-y_{j}\right)^{q}\left(\int_{y_{j-1}}^{1}\left|u^{(k)}(t)\right|^{p} w_{k}(t) d t\right)^{\frac{q}{p}}\right. \\
& \times\left(\int_{x_{j}}^{y_{j}} w_{0}(t) d t\right)\left(\int_{y_{j-1}}^{1} w_{k}^{1-p^{\prime}}(t) d t\right)^{\frac{q}{p^{\prime}}} \\
& +\left(\int_{y_{j}}^{y_{j-1}}\left|u^{(k)}(t)\right|^{p} w_{k}(t) d t\right)^{\frac{q}{p}}\left(\int_{x_{j}}^{y_{j}} w_{0}(t) d t\right) \\
& \left.\times\left(\int_{y_{j}}^{y_{j-1}}\left(t-y_{j}\right)^{(k-1) p^{\prime}} w_{k}^{1-p^{\prime}}(t) d t\right)^{\frac{q}{p^{\prime}}}\right]
\end{aligned}
$$

and condition (19) (with $a=y_{j-1}$ ) leads finally to the estimate

$$
\begin{aligned}
2^{(j-1) q} \int_{x_{j}}^{y_{j}} w_{0}(t) d t \leq & 2^{q-1} c^{q} C^{q}\left(y_{j-1}-y_{j}\right)^{q}\left(\int_{0}^{1}\left|u^{(k)}(t)\right|^{p} w_{k}(t) d t\right)^{\frac{q}{p}} \\
& +2^{q-1} c^{q}\left(\int_{y_{j}}^{y_{j-2}}\left|u^{(k)}(t)\right|^{p} w_{k}(t) d t\right)^{\frac{q}{p}} . \\
& \times\left(\int_{x_{j}}^{y_{j}} w_{0}(t) d t\right)\left(\int_{y_{j}}^{y_{j-1}^{-1}}\left(t-y_{j}\right)^{(k-1) p^{\prime}} w_{k}^{1-p^{\prime}}(t) d t\right)^{\frac{q}{p^{\prime}}} .
\end{aligned}
$$


If

$$
\int_{0}^{x_{j}}\left(x_{j}-t\right)^{(k-1) p^{\prime}} w_{k}^{1-p^{\prime}}(t) d t \leq \int_{y_{j}}^{1}\left(t-y_{j}\right)^{(k-1) p^{\prime}} w_{k}^{1-p^{\prime}}(t) d t,
$$

then according to condition (21) (with $a=x_{j}$ and $b=y_{j}$ ) we have

$$
\left(\int_{0}^{x_{j}}\left(x_{j}-t\right)^{(k-1) p^{\prime}} w_{k}^{1-p^{\prime}}(t) d t\right)^{\frac{q_{p}}{p^{\prime}}}\left(\int_{x_{j}}^{y_{j}} w_{0}(t) d t\right) \leq C^{q}
$$

and (25) yields

$$
\begin{aligned}
& 2^{(j-1) q} \int_{x_{j}}^{y_{j}} w_{0}(t) d t \\
& \quad \leq c_{1}\left[\left(x_{j}-x_{j-1}\right)^{q}\left(\int_{0}^{1}\left|u^{(k)}(t)\right|^{p} w_{k}(t) d t\right)^{\frac{q}{p}}+\left(\int_{x_{j-1}}^{x_{j}}\left|u^{(k)}(t)\right|^{p} w_{k}(t) d t\right)^{\frac{8}{p}}\right] .
\end{aligned}
$$

If in (27) the reverse inequality holds, we have - again according to (21) - that

$$
\left(\int_{y_{j}}^{1}\left(t-y_{j}\right)^{(k-1) q^{\prime}} w_{k}^{1-p^{\prime}}(t) d t\right)^{\frac{q}{p^{\prime}}}\left(\int_{x_{j}}^{y_{j}} w_{0}(t) d t\right) \leq C^{q}
$$

and (26) yields

$$
\begin{aligned}
& 2^{(j-1) q} \int_{x_{j}}^{y_{j}} w_{0}(t) d t \\
& \quad \leq c_{1}\left[\left(y_{j-1}-y_{j}\right)^{q}\left(\int_{0}^{1}\left|u^{(k)}(t)\right|^{p} w_{k}(t) d t\right)^{\frac{g}{p}}+\left(\int_{y_{j}}^{y_{j-1}}\left|u^{(k)}(t)\right|^{p} w_{k}(t) d t\right)^{\frac{q}{p}}\right] .
\end{aligned}
$$

But then we have from (23) that

$$
\begin{aligned}
& \int_{0}^{1}|u(x)|^{q} w_{0}(x) d x \\
& \leq 4 c_{1} \sum_{j \leq m}\left[\left(x_{j}-x_{j-1}\right)^{q}+\left(y_{j-1}-y_{j}\right)^{q}\right]\left(\int_{0}^{1}\left|u^{(k)}(t)\right|^{p} w_{k}(t) d t\right)^{\frac{q}{p}} \\
& \quad+4 c_{1} \sum_{j \leq m}\left[\left(\int_{x_{j-1}}^{x_{j}}\left|u^{(k)}(t)\right|^{p} w_{k}(t) d t\right)^{\frac{g}{p}}+\left(\int_{y_{j}}^{y_{j-1}}\left|u^{(k)}(t)\right|^{p} w_{k}(t) d t\right)^{\frac{q}{p}}\right]
\end{aligned}
$$

and the Hardy inequality (1) follows as $\underset{p}{q} \geq 1$ 


\section{The case II}

Now, let us consider inequality (1) on the class of functions satisfying the conditions

$$
\left.\begin{array}{rl}
u(0)=u^{\prime}(0)=\ldots=u^{(k-1)}(0) & =0 \\
u^{(k-1)}(1) & =0 .
\end{array}\right\}
$$

Similarly as in the Case I, we can derive sufficient conditions combining some welldetermined classes. E.g., the boundedness of the functions $B_{1}, B_{2}$ from (11) or the boundedness of the functions $\widetilde{B}_{1}, \widetilde{B}_{2}$ from (15) is such a sufficient condition, since the boundedness of $B_{1}, B_{2}$ guarantees the validity of (1) for functions satisfying conditions (9), and the boundedness of $\widetilde{B}_{1}, \widetilde{B}_{2}$ guarantees the validity of (1) for functions satisfying conditions (13). Now, conditions (9) together with (13) cover the case (28).

Here, we will proceed in another way. We will reduce the investigation of inequality (1) for functions satisfying (28) to the investigation of a weighted norm inequality for a special (integral) operator. For this purpose, let us introduce, for a fixed number $z$, $0<z<1$, the operator $T_{z}$ by the formula

$$
\left(T_{z} f\right)(x)=\sum_{i=1}^{4}\left(T_{i} f\right)(x)
$$

where

$$
\begin{aligned}
& \left(T_{1} f\right)(x)=\frac{1}{(k-1) !} \chi_{(0, z)}(x) \int_{0}^{x}(x-s)^{k-1} f(s) d s \\
& \left(T_{2} f\right)(x)=\frac{1}{(k-1) !} \chi_{(z, 1)}(x) \int_{0}^{z}\left[(x-s)^{k-1}-(x-z)^{k-1}\right] f(s) d s \\
& \left(T_{3} f\right)(x)=\frac{1}{(k-1) !} \chi_{(z, 1)}(x) \int_{z}^{x}\left[(x-z)^{k-1}-(x-s)^{k-1}\right] f(s) d s \\
& \left(T_{4} f\right)(x)=\frac{1}{(k-1) !} \chi_{(z, 1)}(x) \int_{x}^{1}(x-z)^{k-1} f(s) d s
\end{aligned}
$$

where $\chi(a, b)$ denotes the characteristic function of the interval $(a, b)$. It is easy to see that the operators $T_{i}$ are positive, i.e., that $\left(T_{i} f\right) \geq 0$ for $f \geq 0(i=1, \ldots, 4)$.

Lemma 1. The problem of investigating inequality (1) for functions $u$ satisfying (28) is equivalent to the problem of investigating the weighted norm inequality

$$
\left(\int_{0}^{1}\left|T_{z} f(x)\right|^{q} w_{0}(x) d x\right)^{\frac{1}{q}} \leq c\left(\int_{0}^{1}|f(x)|^{p} w_{k}(x) d x\right)^{\frac{1}{p}}
$$


for functions $f$ satisfying the additional condition

$$
\int_{0}^{z} f(t) d t=\int_{z}^{1} f(t) d t
$$

Proof. (i) Let $u$ satisfy (28). Then we have

$$
u(x)=\frac{1}{(j-1) !} \int_{0}^{x}(x-t)^{j-1} u^{(j)}(t) d t \quad(j=1,2, \ldots, k)
$$

and for any fixed $z, 0<z<1$, we can write

$$
u^{(k-1)}(t)= \begin{cases}\int_{0}^{t} u^{(k)}(s) d s & \text { for } t \leq z \\ -\int_{t}^{1} u^{(k)}(s) d s & \text { for } t \geq z\end{cases}
$$

Using (33) for $x>z$ and $j=k-1$, we obtain after easy calculations that, with (34),

$$
\begin{aligned}
u(x)= & \frac{1}{(k-2) !} \int_{0}^{x}(x-t)^{k-2} u^{(k-1)}(t) d t \\
= & \frac{1}{(k-2) !} \int_{0}^{z}(x-t)^{k-2} \int_{0}^{t} u^{(k)}(s) d s d t-\frac{1}{(k-2) !} \int_{z}^{x}(x-t)^{k-2} \int_{t}^{1} u^{(k)}(s) d s d t \\
= & \frac{1}{(k-1) !}\left\{\int_{0}^{z}\left[(x-s)^{k-1}-(x-z)^{k-1}\right] u^{(k)}(s) d s\right. \\
& \left.-\int_{z}^{x}\left[(x-z)^{k-1}-(x-s)^{k-1}\right] u^{(k)}(s) d s-(x-z)^{k-1} \int_{x}^{1} u^{(k)}(s) d s\right\} .
\end{aligned}
$$

Denoting

$$
f(x)= \begin{cases}u^{(k)}(x) & \text { for } x \leq z \\ -u^{(k)}(x) & \text { for } x>z\end{cases}
$$

we immediately have $u(x)=\left(T_{z} f\right)(x)$. Since $|f(x)|=\left|u^{(k)}(x)\right|$ and $\int_{0}^{z} f(x) d x=$ $\int_{z}^{1} f(x) d x$, we see that (1), (28) imply (31), (32).

(ii) Let $f$ satisfy (31) and (32) and define $F$ as

$$
F(x)= \begin{cases}f(x) & \text { for } x \leq z \\ -f(x) & \text { for } x>z\end{cases}
$$

Then the function $u=T_{z} f$ is a solution of the Cauchy problem

$$
\left.\begin{array}{rl}
u^{(k)}=F & \text { in } \quad(0,1) \\
u^{(i)}(0)=0 & \text { for } i=1,2, \ldots, k-1,
\end{array}\right\}
$$

and due to (32), $u$ satisfies also the condition $u^{(k-1)}(1)=0$. Inequality (1) now follows from (31) 
Remark. The idea of reducing inequality (1) with condition (28) to the weighted norm inequality for the operator $T_{z}$ was submitted to us by Prof. $\mathrm{R}$. Oinarov which is here gratefully acknowledged.

Theorem 2. Let $1<p \leq q<\infty$. Let $w_{0}$ and $w_{k}$ be weight functions on $(0,1)$ and let $z \in(0,1)$ be determined by the condition

$$
\int_{0}^{z} w_{k}^{1-p^{\prime}}(t) d t=\int_{z}^{1} w_{k}^{1-p^{\prime}}(t) d t
$$

Assume that the following numbers are bounded:

$$
\begin{aligned}
& B_{1}=\sup _{0<x<z}\left(\int_{x}^{z}(t-x)^{(k-1) q} w_{0}(t) d t\right)^{\frac{1}{q}}\left(\int_{0}^{x} w_{k}^{1-p^{\prime}}(t) d t\right)^{\frac{1}{p^{\prime}}} \\
& B_{2}=\sup _{0<x<z}\left(\int_{x}^{z} w_{0}(t) d t\right)^{\frac{1}{q}}\left(\int_{0}^{x}(x-t)^{\left.(k-1) p^{\prime} w_{k}^{1-p^{\prime}}(t) d t\right)^{\frac{1}{p^{\prime}}}}\right. \\
& B_{3}=\sup _{z<x<1}\left(\int_{x}^{1}(t-z)^{(k-2) q} w_{0}(t) d t\right)^{\frac{1}{q}}\left(\int_{z}^{x}(t-z)^{p^{\prime}} w_{k}^{1-p^{\prime}}(t) d t\right)^{\frac{1}{p^{\prime}}} \\
& B_{4}=\sup _{z<x<1}\left(\int_{z}^{x}(t-z)^{(k-1) q} w_{0}(t) d t\right)^{\frac{1}{q}}\left(\int_{x}^{1} w_{k}^{1-p^{\prime}}(t) d t\right)^{\frac{1}{p^{\prime}}} \\
& B_{5}=\left(\int_{z}^{1} t^{(k-2) q} w_{0}(t) d t\right)^{\frac{1}{q}}\left(\int_{0}^{z}(z-t)^{p^{\prime}} w_{k}^{1-p^{\prime}}(t) d t\right)^{\frac{1}{p^{\prime}}} .
\end{aligned}
$$

Then the Hardy inequality (1) holds for every function u satisfying conditions (28).

Proof. Due to Lemma 1, it suffices to show that $B_{i}<\infty(i=1, \ldots, 5)$ implies inequality (31) for all $f$ satisfying (32). Inequality (31) can be rewritten by using the notation $\|v\|_{r, w}=\left(\int_{0}^{1}|v(x)|^{r} w(x) d x\right)^{1 / r}$ in the form

$$
\left\|T_{z} f\right\|_{q, w_{0}} \leq c\|f\|_{p, w_{k}} .
$$

(i) The pair of conditions $B_{1}<\infty, B_{2}<\infty$ is necessary and sufficient for the validity of the inequality

$$
\left(\int_{0}^{z} w_{0}(x)\left|\int_{0}^{x}(x-t)^{k-1} f(t) d t\right|^{q} d x\right)^{\frac{1}{q}} \leq c_{1}\left(\int_{0}^{z}|f(t)|^{p} w_{k}(t) d t\right)^{\frac{1}{p}}
$$

(this result is due to V. Stepanov; see, e.g., [3: Section 10]). But the last inequality implies

$$
\left\|T_{1} f\right\|_{q, w_{0}} \leq c_{1}\|f\|_{p, w_{k}}
$$


due to the definition of $T_{1}$ (see (30)).

(ii) The inequality

$$
(x-z)^{k-1}-(x-s)^{k-1} \leq(s-z)(k-1)(x-z)^{k-2}
$$

for $0 \leq z \leq s \leq x$ implies that

$$
\left\|T_{3} f\right\|_{q, w_{0}} \leq c_{2}\left(\int_{z}^{1} w_{0}(x)(x-z)^{(k-2) q}\left|\int_{z}^{z}(s-z) f(s) d s\right|^{q} d x\right)^{\frac{1}{q}}
$$

The condition $B_{3}<\infty$ is necessary and sufficient for the validity of the following Hardy inequality for the function $F(s)=(s-z) f(s)$ :

$$
\begin{aligned}
\left(\int_{z}^{1} W_{0}(x)\left|\int_{z}^{x} F(s) d s\right|^{q} d x\right)^{\frac{1}{q}} & \leq c_{3}\left(\int_{z}^{1}|F(s)|^{p}(s-z)^{-p} w_{k}(s) d s\right)^{\frac{1}{p}} \\
& =c_{3}\left(\int_{z}^{1}|f(s)|^{p} w_{k}(s) d s\right)^{\frac{1}{p}}
\end{aligned}
$$

with $W_{0}(x)=w_{0}(x)(x-z)^{(k-2) q}$, and the last two inequalities imply

$$
\left\|T_{3} f\right\|_{q, w_{0}} \leq c_{4}\|f\|_{p, w_{k}} .
$$

(iii) Similarly, we have

$$
\begin{aligned}
\left\|T_{4} f\right\|_{q, w_{0}} & \leq\left(\int_{z}^{1} w_{0}(x)(x-z)^{(k-1) q}\left|\int_{x}^{1} f(s) d s\right|^{q} d x\right)^{\frac{1}{q}} \\
& \leq c_{5}\left(\int_{z}^{1}|f(s)|^{p} w_{k}(s) d s\right)^{\frac{1}{p}} \\
& \leq c_{5}\|f\|_{p, w_{k}}
\end{aligned}
$$

if and only if $B_{4}<\infty$, since it is in fact a Hardy inequality on $(z, 1)$ with weight $w_{0}(x)(x-z)^{(k-1) q}$ at the left-hand side.

(iv) Using the inequality

$$
(x-s)^{k-1}-(x-z)^{k-1} \leq(z-s)(k-1)(x-s)^{k-2} \leq(z-s)(k-1) x^{k-2}
$$


for $0 \leq s \leq z \leq x$ and then Hölder's inequality, we obtain

$$
\begin{aligned}
\left\|T_{2} f\right\|_{q, w_{0}} \leq & \left(\int_{z}^{1} w_{0}(x)\left|\int_{0}^{z}\left[(x-s)^{k-1}-(x-z)^{k-1}\right] f(s) d s\right|^{q} d x\right)^{\frac{1}{q}} \\
\leq & c_{6}\left(\int_{z}^{1} w_{0}(x) x^{(k-2) q}\left|\int_{0}^{z} f(s)(z-s) d s\right|^{q}\right)^{\frac{1}{q}} \\
= & c_{6}\left(\int_{z}^{1} x^{(k-2) q} w_{0}(x) d x\right)^{\frac{1}{q}}\left|\int_{0}^{z} f(s)(z-s) d s\right| \\
\leq & c_{6}\left(\int_{z}^{1} x^{(k-2) q} w_{0}(x) d x\right)^{\frac{1}{q}} \\
& \times\left(\int_{0}^{z}|f(s)|^{p} w_{k}(x) d x\right)^{\frac{1}{p}}\left(\int_{0}^{z}(z-s)^{p^{\prime}} w_{k}^{1-p^{\prime}}(s) d s\right)^{\frac{1}{p^{\prime}}} \\
= & c_{6} B_{5}\|f\|_{p, w_{k}}
\end{aligned}
$$

provided $B_{5}<\infty$.

(v) Since $\left\|T_{z} f\right\|_{q, w_{0}} \leq \sum_{i=1}^{4}\left\|T_{i} f\right\|_{q, w_{0}}$, the inequality (42) - and consequently, the assertion of Theorem 2 - follows from (43) - (46)

Moreover, some of the conditions $B_{i}<\infty$ are also necessary for (1) to hold with functions $u$ satisfying (28). This follows from the following assertion.

Lemma 2. Let $1<p \leq q<\infty$. Let $w_{0}$ and $w_{k}$ be weight functions and let $z$ be determined by (36). If the inequality (42), i.e. $\left\|T_{x} f\right\|_{q, w_{0}} \leq C\|f\|_{p, w_{k}}$ holds for functions $f$ satisfying (32), then $B_{i}<\infty$ for $i=1,2,3,4$ with $B_{i}$ given by formulas (37) $-(40)$.

Proof. (i) For every $t \in[0, z]$, there is a $t_{1} \in[z, 1]$ such that

$$
\int_{0}^{t} w_{k}^{1-p^{\prime}}(s) d s=\int_{i_{1}}^{1} w_{k}^{1-p^{\prime}}(s) d s .
$$

Define

$$
f_{1}(s)= \begin{cases}w_{k}^{1-p^{\prime}}(s) & \text { for } s \in(0, t) \\ 0 & \text { for } s \in\left[t, t_{1}\right] \\ w_{k}^{1-p^{\prime}}(s) & \text { for } s \in\left(t_{1}, 1\right) .\end{cases}
$$

Then $f_{1}$ satisfies (32), and since $f_{1} \geq 0$ and the operators $T_{i}$ are positive, we have from (42) that

$$
\left\|T_{1} f_{1}\right\|_{g, w_{0}} \leq\left\|T_{z} f_{1}\right\|_{g, w_{0}} \leq C\left\|f_{1}\right\|_{p, w_{k}} .
$$


Now, $\left(w_{k}^{1-p^{\prime}}\right)^{p} w_{k}=w_{k}^{1+p\left(1-p^{\prime}\right)}=w_{k}^{1-p^{\prime}}$ and

$$
\left\|f_{1}\right\|_{p, w_{k}}=\left(\int_{0}^{t} w_{k}^{1-p^{\prime}}(s) d s+\int_{t_{1}}^{1} w_{k}^{1-p^{\prime}}(s) d s\right)^{\frac{1}{p}}=2^{\frac{1}{p}}\left(\int_{0}^{t} w_{k}^{1-p^{\prime}}(s) d s\right)^{\frac{1}{p}}
$$

while

$$
\begin{aligned}
(k-1) !\left\|T_{1} f\right\|_{q, w_{0}} & \geq\left(\int_{0}^{z} w_{0}(x)\left|\int_{0}^{x}(x-s)^{k-1} f_{1}(s) d s\right|^{q} d x\right)^{\frac{1}{q}} \\
& \geq\left(\int_{t}^{z} w_{0}(x)\left(\int_{0}^{x}(x-s)^{k-1} f_{1}(s) d s\right)^{q} d x\right)^{\frac{1}{q}} \\
& =\left(\int_{t}^{z} w_{0}(x)\left(\int_{0}^{t}(x-s)^{k-1} w_{k}^{1-p^{\prime}}(s) d s\right)^{q} d x\right)^{\frac{1}{q}} \\
& \geq\left(\int_{t}^{z} w_{0}(x)\left(\int_{0}^{t}(x-t)^{k-1} w_{k}^{1-p^{\prime}}(s) d s\right)^{q} d x\right)^{\frac{1}{q}} \\
& =\left(\int_{t}^{z} w_{0}(x)(x-t)^{(k-1) q} d x\right)^{\frac{1}{q}}\left(\int_{0}^{t} w_{k}^{1-p^{\prime}}(s) d s\right) .
\end{aligned}
$$

From (49), we obtain

$$
\left(\int_{t}^{z}(x-t)^{(k-1) q} w_{0}(x) d x\right)^{\frac{1}{q}}\left(\int_{0}^{t} w_{k}^{1-p^{\prime}}(s) d s\right) \leq C 2^{\frac{1}{p}}\left(\int_{0}^{t} w_{k}^{1-p^{\prime}}(s) d s\right)^{\frac{1}{p}}
$$

and since $t \in(0, z)$ was arbitrary, we have $B_{1} \leq C 2^{\frac{1}{p}}$.

(ii) For the same function $f_{1}$ from (48), we have

$$
\left\|f_{1}\right\|_{p, w_{k}}=2^{\frac{1}{p}}\left(\int_{i_{1}}^{1} w_{k}^{1-p^{\prime}}(s) d s\right)^{\frac{1}{p}}
$$

and

$$
(k-1) !\left\|T_{4} f_{1}\right\|_{q, w_{0}}=\left(\int_{z}^{1} w_{0}(x)(x-z)^{(k-1) q}\left(\int_{x}^{1} f_{1}(s) d s\right)^{q} d x\right)^{\frac{1}{q}}
$$




$$
\begin{aligned}
& \geq\left(\int_{z}^{t_{1}}(x-z)^{(k-1) q} w_{0}(x)\left(\int_{t_{1}}^{1} w_{k}^{1-p^{\prime}}(s) d s\right)^{q} d x\right)^{\frac{1}{q}} \\
& =\left(\int_{z}^{t_{1}}(x-z)^{(k-1) q} w_{0}(x) d x\right)^{\frac{1}{q}} \cdot\left(\int_{t_{1}}^{1} w_{k}^{1-p^{\prime}}(s) d s\right)
\end{aligned}
$$

and the inequality $\left\|T_{4} f_{1}\right\|_{q, w_{0}} \leq C\left\|f_{1}\right\|_{p, w_{k}}$ which follows from (42) implies that

$$
\left(\int_{z}^{t_{1}}(x-z)^{(k-1) q} w_{0}(x) d x\right)^{\frac{1}{q}}\left(\int_{t_{1}}^{1} w_{k}^{1-p^{\prime}}(s) d s\right) \leq C 2^{\frac{1}{p}}\left(\int_{t_{1}}^{1} w_{k}^{1-p^{\prime}}(s) d s\right)^{\frac{1}{p}}
$$

and since $t_{1} \in(z, 1)$ was arbitrary, we have $B_{4} \leq C 2^{\frac{1}{p}}$.

(iii) Let $t$ and $t_{1}$ be given by (47) and define

$$
f_{2}(s)= \begin{cases}(t-s)^{(k-1)\left(p^{\prime}-1\right)} w_{k}^{1-p^{\prime}}(s) & \text { for } 0<s<t \\ 0 & \text { for } t \leq s \leq t_{1} \\ \tilde{c} w_{k}^{1-p^{\prime}}(s) & \text { for } t_{1}<s<1\end{cases}
$$

where $\tilde{c}$ is choosen so that

$$
\int_{0}^{\ell}(t-s)^{(k-1)\left(p^{\prime}-1\right)} w_{k}^{1-p^{\prime}}(s) d s=\tilde{c} \int_{i_{1}}^{1} w_{k}^{1-p^{\prime}}(s) d s .
$$

Then $f_{2}$ satisfies (32) and the Hölder inequality yields

$$
\begin{aligned}
\int_{0}^{t}(t-s)^{(k-1)\left(p^{\prime}-1\right)} w_{k}^{1-p^{\prime}}(s) d s \\
\quad=\int_{0}^{t}(t-s)^{(k-1)\left(p^{\prime}-1\right)} w_{k}^{\left(1-p^{\prime}\right) / p}(s) w_{k}^{\left(1-p^{\prime}\right) / p^{\prime}}(s) d s \\
\quad \leq\left(\int_{0}^{t}(t-s)^{(k-1) p^{\prime}} w_{k}^{1-p^{\prime}}(s) d s\right)^{\frac{1}{p}}\left(\int_{0}^{t} w_{k}^{1-p^{\prime}}(s) d s\right)^{\frac{1}{p^{\prime}}}
\end{aligned}
$$

i.e., due to (47),

$$
\int_{0}^{t}(t-s)^{(k-1) p^{\prime}} w_{k}^{1-p^{\prime}}(s) d s \geq \tilde{c}^{p} \int_{t_{1}}^{1} w_{k}^{1-p^{\prime}}(s) d s
$$


Now,

$$
\begin{aligned}
\left\|f_{2}\right\|_{p, w_{k}} & =\left(\int_{0}^{t}(t-s)^{(k-1) p^{\prime}} w_{k}^{1-p^{\prime}}(s) d s+\tilde{c}^{p} \int_{\ell_{1}}^{1} w_{k}^{1-p^{\prime}}(s) d s\right)^{\frac{1}{p}} \\
& \leq 2^{\frac{1}{p}}\left(\int_{0}^{t}(t-s)^{(k-1) p^{\prime}} w_{k}^{1-p^{\prime}}(s) d s\right)^{\frac{1}{p^{\prime}}}
\end{aligned}
$$

and

$$
\begin{aligned}
(k-1) !\left\|T_{1} f_{2}\right\|_{q, w_{0}} & =\left(\int_{0}^{z}\left(\int_{0}^{x}(x-s)^{k-1} f_{2}(s) d s\right)^{q} w_{0}(x) d x\right)^{\frac{1}{q}} \\
& \geq\left(\int_{t}^{z} w_{0}(x)\left(\int_{0}^{t}(x-s)^{k-1}(t-s)^{(k-1)\left(p^{\prime}-1\right)} w_{k}^{1-p^{\prime}}(s) d s\right)^{q} d x\right)^{\frac{1}{q}} \\
& \geq\left(\int_{t}^{z} w_{0}(x) d x\right)^{\frac{1}{q}}\left(\int_{0}^{t}(t-s)^{(k-1) p^{\prime}} w_{k}^{1-p^{\prime}}(s) d s\right) .
\end{aligned}
$$

The inequality $\left\|T_{1} f_{2}\right\|_{q, w_{0}} \leq C\left\|f_{2}\right\|_{p, w_{k}}$ yields finally $B_{2} \leq C 2^{\frac{1}{p}}$.

(iv) For $t$ and $t_{1}$ given by (47), define

$$
f_{3}(s)= \begin{cases}\tilde{c} w_{k}^{1-p^{\prime}}(s) & \text { for } t<s<z \\ (s-z)^{p^{\prime}-1} w_{k}^{1-p^{\prime}}(s) & \text { for } z<s<t_{1} \\ 0 & \text { otherwise in }(0,1)\end{cases}
$$

where $\tilde{c}$ is choosen so that

$$
\int_{z}^{t_{1}}(s-z)^{p^{\prime}-1} w_{k}^{1-p^{\prime}}(s) d s=\tilde{c} \int_{t}^{z} w_{k}^{1-p^{\prime}}(s) d s .
$$

Then $f_{3}$ satisfies (32), and similarly as in part (iii), the Hölder inequality yields

$$
\int_{z}^{t_{1}}(s-z)^{p^{\prime}} w_{k}^{1-p^{\prime}}(s) d s \geq \tilde{c}^{p} \int_{t}^{z} w_{k}^{1-p^{\prime}}(s) d s .
$$

Now,

$$
\begin{aligned}
\left\|f_{3}\right\|_{p, w_{k}} & =\left(\int_{z}^{t_{1}}(s-z)^{p^{\prime}} w_{k}^{1-p^{\prime}}(s) d s+\tilde{c}^{p} \int_{t}^{z} w_{k}^{1-p^{\prime}}(s) d s\right)^{\frac{1}{p}} \\
& \leq 2^{\frac{1}{p}}\left(\int_{z}^{t_{1}}(s-z)^{p^{\prime}} w_{k}^{1-p^{\prime}}(s) d s\right)^{\frac{1}{p}}
\end{aligned}
$$


and, due to the inequality $(x-z)^{k-1}-(x-s)^{k-1} \geq(s-z)(x-z)^{k-2}$,

$$
\begin{aligned}
(k-1) !\left\|T_{3} f_{3}\right\|_{q, w_{0}} & \geq\left(\int_{z}^{1} w_{0}(x)(x-z)^{k-2) q}\left|\int_{z}^{x}(s-z) f_{3}(s) d s\right|^{q} d x\right)^{\frac{1}{q}} \\
& \geq\left(\int_{t_{1}}^{1} w_{0}(x)(x-z)^{k-2) q}\left(\int_{z}^{t_{1}}(s-z)^{p^{\prime}} w_{k}^{1-p^{\prime}}(s) d s\right)^{q} d x\right)^{\frac{1}{q}} \\
& =\left(\int_{l_{1}}^{1} w_{0}(x)(x-z)^{(k-2) q} d x\right)^{\frac{1}{q}}\left(\int_{z}^{t_{1}}(s-z)^{p^{\prime}} w_{k}^{1-p^{\prime}}(s) d s\right) .
\end{aligned}
$$

The inequality $\left\|T_{3} f_{3}\right\|_{q, w_{0}} \leq C\left\|f_{3}\right\|_{p, w_{k}}$ yields finally $B_{3} \leq C 2^{\frac{1}{p}}$

Acknowledgement. This paper was written while the first author was visiting University of Bayreuth which is here gratefully acknowledged. His research was also partially supported by the Grant Agency of Czech Republic, Grant No. 201/94/1066.

\section{References}

[1] Drábek, P. and A. Kufner: The Hardy inequality and Birkhoff interpolation. Bayreuther Math. Schriften 47 (1994), 99 - 104.

[2] Kufner, A.: Higher order Hardy inequalities. Bayreuther Math. Schriften 44 (1993), 105 $-146$.

[3] Opic, B. and A. Kufner: Hardy-Type Inequalities. Harlow: Longman Sci. \& Techn. 1990.

[4] Sinnamon, G.: Kufner's conjecture for higher order Hardy inequalities. Real Analysis Exchange $21(1995 / 96), 590-601$.

Received 30.07.1996; in revised form 24.10.1996 